ISSN 2693-2504

\title{
New Surgical Procedure to Re-Start the Hearth after Open Heart Surgery, Reducing Post-Op Cardiac Distress
}

Journal of Bioscience \& Biomedical Engineering

Original Research

Michael Burgio

Burgio Enterprises Ltd., Palm Bay, Florida 32905, USA

\author{
*Correspondence author \\ Michael Burgio \\ Burgio Enterprises Ltd. \\ Palm Bay \\ Florida 32905 \\ USA
}

Submitted : 29 July 2020 ; Published : 20 Aug 2020

\begin{abstract}
I developed this surgical procedure during the time I had the privilege of working with Dr. Michael DeBakey. My concern in using paddles to restart the heart had the potential of significant problems postop. As an example, when the panels are placed into the chest cavity one lies directly over the left descending artery. Which supplies one-third of the blood to the heart. Any inflammation of the artery has the potential of creating an additional cardiac event. In developing this procedure, I made every effort to simplify the procedure. There only three plates, a catheter coated with silicone, a standard surgical needle, and a cardiac pacemaker analyzer. In addition to the potential inflammation, when the panels are inserted in the chest cavity the heart is stimulated to a rate of something close to $72 \mathrm{bpm}$. The difficulty is in most cases the chambers have a minimal amount of blood. Creating a distinct possibility of damaging the internal walls. Using this procedure, we are able to start the heart beating at a rate as low as $5 \mathrm{bpm}$ and increase the rate in direct proportion to the blood flow from the heart-lung machine. As the blood is filling the chambers the rate is increased proportionately. Reducing the possibility of damage to the internal walls of all four chambers. In addition, this method can independently control, the amplitude, the pulse width, the AV delay, and the rate. Prior to this surgical procedure many patients were denied openheart surgery. They were too sick, and a successful outcome was in question. Open-heart surgeries is now available to the sickest of the sick, the elderly, and the patients with so many complications due to years of a lack of quality healthcare.

Once the surgical procedure is completed and the chest cavity is closed the catheters are left in myocardial tissue, to be utilized when the patient arrives in ICU, Surgical ICU. In the critical care unit, the catheters are connected to an external pacemaker attached to the patient. Because the catheters are within the myocardium there can be no loss of capture or stimulation. This allows a number of options if the patient goes into distress. Some of the variables within the external pacemaker, the AV-RV pathway through the bundle of His can be extended or shortened to control Wolff Parkinson's White Syndrome, Ventricular Tachycardia, Atrial Tachycardia, Supraventricular Tachycardia, a Cardiac Pause, an Internal EKG, and an Internal Defibrillator. If the patient codes the external pacemaker and the catheters could be program as a Dual Chamber (AV) Fixed Rate Pacemaker, providing total control and reducing the possibility of losing the patient. If the patient once again goes into a cardiac arrest, the external pacemaker parameters can be adjusted to increase the Amplitude, Rate, and Pulse Width and retuning to a normal sinus rhythm. Additional cardiac events, Heart Failure, Irregular Rhythm, Premature Ventricular Contractions (PVC's) Atrioventricular Block, Bundle Branch Block. This is a small sample of the benefits.
\end{abstract}

\section{Assumptions}

Controlling normal sinus rhythm with artificial stimulation, with a pacemaker analyzer. Controlled by three essential elements.

1. Rate. This is nothing more than an ideal number of cardiac contractions per minute, normally $72 \mathrm{bpm}$.

2. Pulse-Width/Amplitude. Pulse-With is the length of time in milliseconds the current is flowing into the cardiac tissue, creating contraction, and depolarization.

3. Atrial-Ventricular Delay. The AV delay is the length of time in milliseconds it takes for the stimulation from the SA node to pass through the Bundle of His through the bifurcation and stimulate the Right Ventricle; RV, causing a contraction. 
Counter-Indications to cardiac paddles used to stimulate the heart after open-heart surgery.

1. When surgery is completed, the object is to re-stimulated heart and redirect blood flow from the heart-lung machine back into the chambers of the heart. Creating normal sinus rhythm, and normal function.

2. The first problem with this method is when the paddles are introduced into the chest cavity, depending on the position of the surgeon. Assuming the surgeon is on the left side of the patient. The panel in his right hand will be introduced to the chest cavity and be placed directly over left sending cardiac artery.

The panel in his left hand will be introduced and will be on the right side of the heart.

By stimulating the heart with these paddles there are three significant problems.

1. The electrical current from the paddles will shock the left descending, creating a reaction generally significant inflammation. It can also create some significant damage to the artery. The inflammation can cause an inclusion to the artery thereby restricting the blood flow and reducing the cardiac output from the right ventricle.

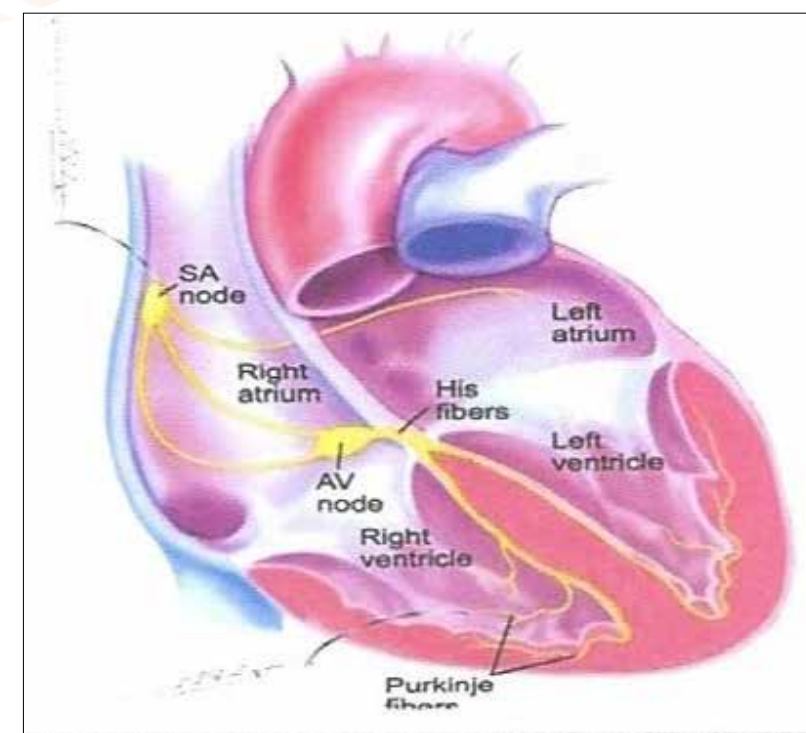

What we have done is to use the advantages of a cardiac pacemaker analyzer. The analyzer has within its functions the ability to control, rate, pulse width, and A V delay. These are critical function in controlling the heart. Providing a controlled blood flow to fill up the chambers, and monitor the connection points of the bypass surgery, to ensure there is no leakage. The most dangers thing to the heart is to start immediately at 72 bpm with a dry chamber. With this procedure we can start the heart beating at a rate of five beats a minute and increase it proportionately to the reverse of the blood flow from the heartlung machine. Avoiding any potential problems to the interior wall of all four chambers.
2. Coordinating the stimulating of the heart with paddles and redirecting the flow from the heart-lung machine back into the heart chambers is an extraordinarily differcult maneuver. The difficulty is when the surgeon and the technician controlling the heart lung machine must be an in absolute sequential timing. If the heart begins to stimulate too quickly with a lack of significant blood within the chambers, the heart is then beating dry.

3. This can cause significant damage to the interior wall particularly of the right ventricle. Potentially scarring, cracking, or weakening the muscle of the RV. The right ventricle's primary responsibility is to contract and forced the blood coming from the A V node into the pulmonary artery and starting circulation. The quality, strength, and the effectiveness of the right ventricle are critical to normal body functions.

\section{The Procedure}

In order to find the best location to stimulate the SA node. The tip of the surgical needle is placed at various locations in the approx. area of the SA node. Keeping a close eye on the monitor with EKG response will help find the closest point to the center of the SA node. Providing the control by stimulating the heart and redirecting the blood flow from the heart-lung machine to the heart chambers at a controlled rate. The two catheters are entered into the myocardium, at the SA Node, and base of the Right Ventricle.
Controlling early contractions or retrograde conduction by setting the A V delay to a very short interval, approximately $125-150 \mathrm{~ms}$. Along with a lengthening of the pulse with. After stimulation the heart will go into an absolute refractory period. In absolute refractory it cannot sense any current stimulation. Without that stimulation there will be no reaction. If there is no reaction, there can be no contraction when the right ventricle is only partially filled.

This method reduces if not eliminated any potential damage to the left descending artery. We have reduced any possibility of scarring/weakening the internal wall of all four chambers. 
We have accomplished a smooth transition from the heartlung machine to normal heart functions. Finally, re-starting the heart is in absolute control during this entire period. Also given the opportunity to closely examine all of the connections at the point of the bypass. Assuring there is no leakage. If the heart is being stimulated at a rate of $5 \mathrm{bpm}$ it is well within the realm of possibility, if there is leakage at anyone of the connections of the bypass, or from heart valve repair, to total heart valve replacement. The leakage will become obvious and additional sutures can be provided to stop the flow. This can be done without interruption from the transition of heart-lung machine to normal heart function.

The catheters are left in the myocardium at the completion of the surgery and extends outside of the chest. Plugged into an external cardiac pacemaker. The pacemaker is generally attached to the patient's arm at the bicep. If there is a cardiac event at any time post-op the nurse simply walks to the patient's bedside and start the external pacemaker. It will begin pacing at a predetermined rate. This will put the patient into normal sinus rhythm. A voiding the potential, indeed any lifethreatening events.

The configuration of the catheter itself has been described, as an according type configuration. That according effect is the anchor to secure the catheter to the heart. When the patient is ready for discharge the catheter is withdrawn so that the tip of the catheter is inside the heart tissue. Providing not only stimulation but if necessary defibrillation. At the time the catheter is to be withdrawn generally $48-72$ hours post-op. When the catheter is fully removed from the heart tissue, the healing will be almost immediate with no blood lost, and little to no damage to the heart tissue.

Prior to this surgical procedure many patients were denied openheart surgery. They were too sick, and a successful outcome was in question. Open-heart surgeries is now available to the sickest of the sick, the elderly, and the patients with so many complications due to years of a lack of quality healthcare.

The catheters that were placed in the myocardium of the heart at the end of the surgery, to restart the heart. Are now connected to a temporary external pacemaker at the bedside. If the heart stops the nurse simply presses the on button and the patient will be in normal sinus rhythm. This allows complete control of pacing and possible use of an internal to the bill later at the bedside. Generally used 24 to 72 hours postop.

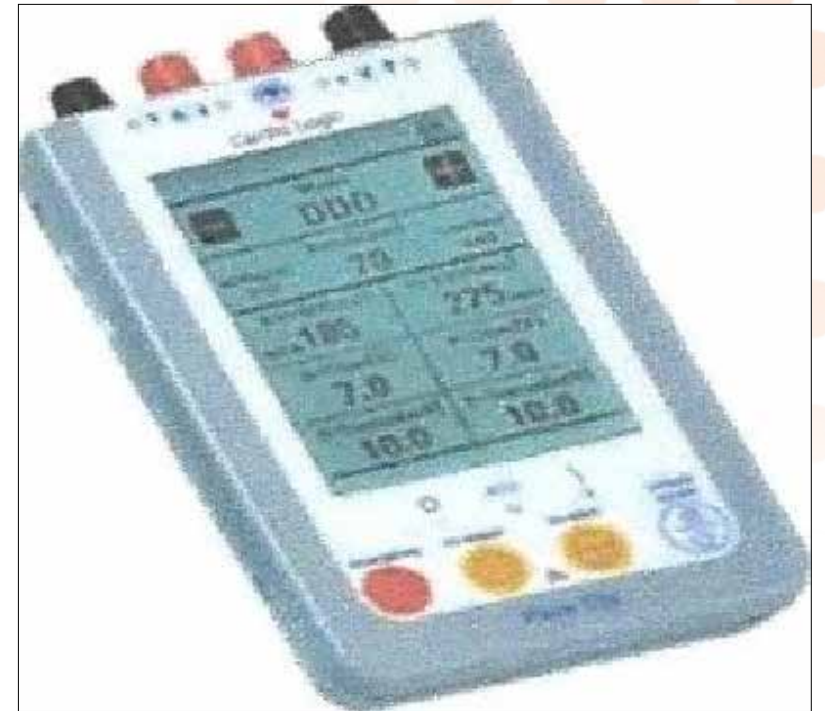

Copyright: (C2020 Michael Burgio. This is an open-access article distributed under the terms of the Creative Commons Attribution License, which permits unrestricted use, distribution, and reproduction in any medium, provided the original author and source are credited. 